\title{
Aditivo para fabricación de ladrillos de yeso: macizos, huecos o semihuecos mediante vibrocompresión
}

\author{
Flores Yepes, José A¹. Berná Serna, Juan Manuel². Gimeno Blanes, Francisco Javier. Pastor \\ Perez, Joaquín¹. Martínez Gabarrón, Antonio'. Luis M. Serna Jara³ \\ 1 Profesor Universidad Miguel Hernández, Elche, Alicante, Spain. Carretera de Beniel, Km. 3,2 \\ 2 Dr. Ingeniero Agrónomo. \\ 3 Ingeniero Técnico Industrial, Grado en Ingeniería Mecánica
}

Resumen: El departamento de Ingeniería de la Universidad Miguel Hernández del Campus de Orihuela lleva más de catorce años investigando sobre el desarrollo de nuevos materiales, la forma de aditivarlos y su aplicación a la sociedad. El aditivo está patentado, es un fluidificante, superplastificante y retardante del fraguado del yeso, compuesto por dióxido de silicio coloidal más ácido cítrico, con proporciones de 1-3 \% de dióxido de silicio y de 99-97 \% de ácido cítrico. Se puede adicionar tanto en fase sólida como en líquida, actuando sobre el fraguado. Es de aplicación en yeso blanco, yeso negro o moreno (Black paster) y escayola. Las dosificaciones, por $\mathrm{kg}$ de yeso, están entre $0.06 \mathrm{gr} / \mathrm{kg}$ hasta $1,2 \mathrm{gr} / \mathrm{kg}$ de yeso, con unos tiempos de fraguado entre 15 y 240 minutos. La dosificación del agua para el amasado es variable, siendo una ventaja la reducción de agua en el masado. Se establece en 0,2 kg de agua por $1 \mathrm{~kg}$ de yeso. Así se obtiene un material que convertido en pequeñas bolas (diámetro entre 2 y $8 \mathrm{~mm}$ ) es utilizable en la fabricación de prefabricados mediante prensado. En la fabricación de ladrillos de yeso se produce una reducción de emisiones de $\mathrm{CO}_{2}$ en torno al $80 \%$, con temperatura de calcinación de unos $250{ }^{\circ} \mathrm{C}$, según el tipo de yeso a conseguir. Se evita el tiempo de secado del ladrillo cerámico (unas 8 horas a $100{ }^{\circ} \mathrm{C}$ ) más el de cocción del mismo (unas 8-14 horas a $900^{\circ} \mathrm{C}$ ). El material tiene un uso industrial en la fabricación de ladrillos huecos o semihuecos (comportamiento a Compresión de unos $19,2323 \mathrm{~N} / \mathrm{mm}^{2}$ ), además de un beneficio medioambiental y económico.

Palabras clave: sulfato cálcico, eficiencia energética, fraguado, ladrillo cerámico, compresión.

\section{Introducción}

Se analiza en el estudio dos líneas de trabajo distintas. Una de ellas, la propia fabricación de ladrillos y sus correspondientes ensayos mecánicos referidos al más concluyente que es la resistencia compresión. La otra, se refiere al estudio energético, o eficiencia del proceso propuesto.

El desarrollo, centrado en la aplicación del sulfato cálcico al desarrollo de elementos prefabricados, se fundamenta en el hecho de sustituir el procedimiento de colada directa por el de prensado o vibro-comprimido habitual en elementos prefabricados tipo bloques, bovedillas, bordillos, adoquines, etc. El intento de fabricación de ladillos por extrusión ha sido, en nuestro caso, infructuoso por disgregarse el agua de la matriz de yeso y producir un emboce en el cuello de salida de la extrusora. No quiere decir esto que no se pueda experimentar en esa línea, si bien, el resultado de la fabricación mediante prensa, por el contrario, sí ha resultado idóneo.

Otro aspecto a reflejar en el estudio es la necesidad de obtener un material "granuloso" que sea capaz de llenar los huecos de un molde, con la suficiente consistencia mecánica, tiempo de 


\section{CONGRESO IBÉRICO DE AGROINGENIERÍA \\ X CONGRESSO IBÉRICO DE AGROENGENHARIA \\ 3 - 6 septiembre 2019, Huesca - España}

fraguado suficiente, plasticidad para moldeo y no adherencia para poder desmoldar. Esta característica que en el amasado de hormigones semisecos es "fácil de conseguir", no lo es tanto cuando se trata de yesos. Para ello, se han elaborado dos procedimientos: uno con aportación de arena (sílice fina tipo arena de playa), y el segundo, que evita la adicción de árido objeto de este estudio.

Para conseguir todo lo descrito, se ha reducido la cantidad de agua de masado a $200 \mathrm{~g}$ por cada $1000 \mathrm{~g}$ de sulfato cálcico estándar de construcción o yeso negro. Se ha aditivado, antes de comenzar el proceso de amasado, con $0.6 \mathrm{~g}$ de aditivo mezclado en la matriz de yeso (también se pude dispersar en agua) y posteriormente se ha amasado con una batidora manual.

En relación a la parte energética, se analiza la parte correspondiente a la fabricación y la de secado o cocción.

El objetivo del estudio que se presenta es mostrar los resultados del proceso de fabricación y de la comparativa energética del ladrillo de sulfato cálcico que se obtiene, con el correspondiente ladrillo cerámico.

\section{Materiales y métodos}

\subsection{Materiales}

Los materiales empleados para la fabricación son los siguientes:

El agua, es uno de los componentes básicos para la formación de la pasta; la empleada en los ensayos es agua de la red de suministro, sin ningún tratamiento especial. Hay que tener en cuenta la temperatura a la que se adiciona, ya que ésta afecta en la cantidad de sulfato de calcio que se disuelve. La empleada en laboratorio ha sido de $25^{\circ} \mathrm{C}$. La tabla 1 , expone los parámetros analíticos del agua empleada.

Tabla 1: Parámetros analíticos del agua empleada en la elaboración de los morteros. (Aquagest Levante, S.A.).

\begin{tabular}{ccc}
\hline Parámetros & Resultados \% & Unidades \\
\hline Amonio & $<0.10 \pm 15$ & $\mathrm{mg} / \mathrm{L}$ \\
Carbono orgánico total & $1.8 \pm 15$ & $\mathrm{mg} / \mathrm{L}$ \\
Cianuros totales & $<5 \pm 28$ & $\mu \mathrm{g} / \mathrm{L}$ \\
Indice de Langelier & 0,46 & - \\
Bicarbonatos & $156.0 \pm 12$ & $\mathrm{mg} / \mathrm{L}$ \\
Calcio & $96.4 \pm 12$ & $\mathrm{mg} / \mathrm{L}$ \\
Carbonatos & $<2.0 \pm 13$ & $\mathrm{mg} / \mathrm{L}$ \\
Conductividad a $20^{\circ} \mathrm{C}$ & $931 \pm 12$ & $\mu \mathrm{cm}$ \\
pH & $7.9 \pm 0.1$ & $\mathrm{U} \cdot \mathrm{pH}$. \\
Sodio & $54.7 \pm 12$ & $\mathrm{mg} / \mathrm{L}$ \\
Cloruros & $90.0 \pm 13.0$ & $\mathrm{mg} / \mathrm{L}$ \\
Fluoruros & $0.150 \pm 12.9$ & $\mathrm{mg} / \mathrm{L}$ \\
Nitratos & $2.6 \pm 13.1$ & $\mathrm{mg} / \mathrm{L}$ \\
Sulfatos & $270.8 \pm 13.1$ & $\mathrm{mg} / \mathrm{L}$ \\
\hline
\end{tabular}

Se ha utilizado Sulfato cálcico para el desarrollo de los ensayos cuyas características nominales vienen definidas según la Norma EN UNE 13279-1, [1], empleando el tipo B1 denominación de Yeso para construcción, siendo las siguientes: Yeso grueso constituido por semihidrato $\left(\mathrm{SO}_{4} \mathrm{Ca} .1 / 2 \mathrm{H}_{2} \mathrm{O}\right)$ con más del $50 \%$ de hemihidrato, con la posible incorporación de aditivos reguladores del fraguado, con una resistencia mecánica mínima a Flexión de $1 \mathrm{~N} / \mathrm{mm}^{2}$ y de $2 \mathrm{~N} / \mathrm{mm}^{2}$ a Compresión (Tabla 2). Este tipo de yeso se utiliza para pasta de agarre en la 


\section{CONGRESO IBÉRICO DE AGROINGENIERÍA \\ X CONGRESSO IBÉRICO DE AGROENGENHARIA \\ 3 - 6 septiembre 2019, Huesca - España}

ejecución de tabicados, en revestimientos interiores, como conglomerante auxiliar de obra y es empleado, además, para prefabricados.

Tabla 2. Especificaciones para los yesos de construcción (Fuente: Norma UNE EN 13279-1) [1].

\begin{tabular}{|c|c|c|c|c|c|c|c|}
\hline \multirow{2}{*}{$\begin{array}{l}\text { Plaster for } \\
\text { construction }\end{array}$} & \multirow{2}{*}{$\begin{array}{l}\text { Binder } \\
\text { plaster } \\
\% \\
\text { content }\end{array}$} & \multicolumn{2}{|c|}{$\begin{array}{c}\text { Principle of setting time } \\
\text { min }\end{array}$} & \multirow{2}{*}{$\begin{array}{c}\text { Flexural } \\
\text { strength } \\
\mathrm{N} / \mathrm{mm}^{2}\end{array}$} & \multirow{2}{*}{$\begin{array}{c}\text { Resistance } \\
\text { to } \\
\text { compression } \\
\mathrm{N} / \mathrm{mm}^{2}\end{array}$} & \multirow{2}{*}{$\begin{array}{c}\text { Surface } \\
\text { hardness } \\
\mathrm{N} / \mathbf{m m}^{2}\end{array}$} & \multirow{2}{*}{$\begin{array}{l}\text { Adhesion } \\
\mathrm{N} / \mathbf{m m}^{2}\end{array}$} \\
\hline & & $\begin{array}{c}\text { Normal } \\
\text { application } \\
\text { plaster }\end{array}$ & $\begin{array}{c}\text { Plaster } \\
\text { projection } \\
\text { mechanics }\end{array}$ & & & & \\
\hline B1 & $\geq 50$ & & & & & & The break \\
\hline B2 & $<50$ & & & & & & occurs on the \\
\hline B3 & a & & & & & & stand or the \\
\hline B4 & $\geq 50$ & & & & & & mass of plaster; \\
\hline B5 & $<50$ & & & & & & $\begin{array}{l}\text { When the break } \\
\text { appears in the }\end{array}$ \\
\hline B6 & a & $>20 \mathrm{~b}$ & $>50$ & $\geq 1,0$ & $\geq 2,0$ & - & $\begin{array}{c}\text { plaster support } \\
\text { interface must } \\
\text { be } \geq 1\end{array}$ \\
\hline B7 & $\geq 50$ & & & $\geq 2,0$ & $\geq 6,0$ & $\geq 2,5$ & \\
\hline
\end{tabular}

a According to paragraphs 3.3, 3.4, 3.5 y 3.6

b Some handheld applications allow one value less than $20 \mathrm{~min}$. In this case, the producer must declare the principle of setting time.

Aditivo para yesos patentado con número de registro P201700812. El aditivo se trata de fluidifante superplastificante y retardardante del fraguado compuesto por dióxido de silicio coloidal más ácido cítrico (o ácido policarboxílico), con proporciones 1-3\% de dióxido de silicio y 99-97\% de ácido cítrico molido y tamizado.

La dosificación realizada en los ensayos ha sido: 1000gr de yeso, 200gr de agua y 0.06gr de aditivo.

\subsection{Procedimientos o métodos}

Proceso de trabajo. Se dosifica en el yeso seco el aditivo sólido y se mezcla durante cinco minutos. Posteriormente se vierte el agua sobre el yeso aditivado y se mezcla con una batidora manual hasta conseguir la dispersión, en forma de bolas, de entre $1-4 \mathrm{~mm}$ de diámetro aproximadamente. Se vierte sobre la máquina de vibrocompresión con un molde semimazizo o ladillo perforado, del mismo modo que se fabrican los bloques o bovedillas. La presión de trabajo de dicha prensa es de $4,5 \mathrm{~kg} / \mathrm{cm}^{2}$.

Las dimensiones de los ladrillos se han fijado en $221 \times 118 \times 45 \mathrm{~mm}$. Se han realizado un total de 10 probetas correspondientes a un solo lote de fabricación.

Una vez obtenido el ladillo se deja secar durante 14 días a temperatura superior a $15^{\circ} \mathrm{C}$ y humedad relativa inferior o igual al $65 \%$ hasta alcanzar el estado de masa constante. Posterior secado de probetas durante 24 horas a $105^{\circ} \mathrm{C}$ con margen de $5^{\circ} \mathrm{C}$, y posterior enfriamiento de 4 horas. Posteriormente se ensaya a compresión en una maquina universal de ensayos. La norma considerada ha sido la UNE-EN 772-1, 2011. [4] 


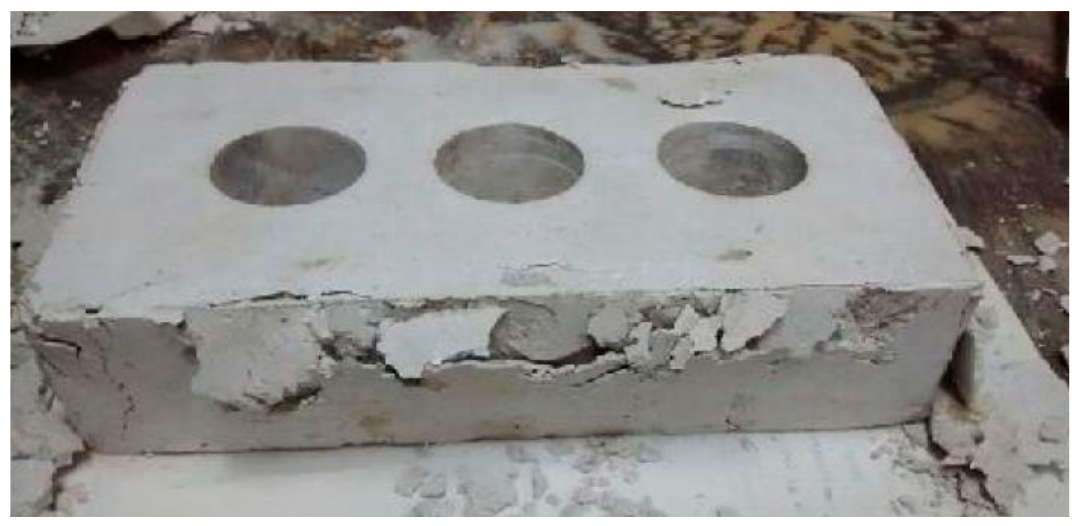

Figura 1. Ladrillo tipo de yeso ensayado.

Con relación al estudio energético. Se evalúa el proceso se fabricación del ladillo de yeso aditivado comparándolo con el cerámico perforado.

\section{Resultados y discusión}

\subsection{Resultados y Discusión resistencia mecánica.}

Los resultados de la rotura de probetas, se muestran en la tabla adjunta, además de los parámetros estadísticos más representativos.

Tabla 3: Resultados de los distintos ensayos.

\begin{tabular}{cccccc}
\hline $\begin{array}{c}\text { Prob } \\
\text { eta }\end{array}$ & $\begin{array}{c}\text { Área Media } \\
\left(\mathrm{mm}^{2}\right)\end{array}$ & $\begin{array}{c}\text { Carga de } \\
\text { rotura }(\mathrm{kN})\end{array}$ & $\begin{array}{c}\text { Resistencia a } \\
\text { Compresión }\left(\mathrm{N} / \mathrm{mm}^{2}\right)\end{array}$ & \multicolumn{2}{c}{$\begin{array}{c}\text { Valores estadísticos de la } \\
\text { variable Compresión }\end{array}$} \\
\hline 1 & 26078 & 581,54 & 22,3 & Media & 19,87 \\
2 & 26078 & 526,78 & 20,2 & Varianza & 4,78 \\
3 & 26078 & 396,39 & 15,2 & Desv. Estand & 2,19 \\
4 & 26078 & 482,44 & 18,5 & Coef Variación & 0,11 \\
5 & 26078 & 524,17 & 20,1 & Xmin & 15,20 \\
6 & 26078 & 466,80 & 17,9 & Cuartil 1 & 18,80 \\
7 & 26078 & 560,68 & 21,5 & Cuartil 2 & 20,15 \\
8 & 26078 & 576,32 & 22,1 & Cuartil 3 & 21,43 \\
9 & 26078 & 513,74 & 19,7 & Mediana & 20,15 \\
10 & 26078 & 552,85 & 21,2 & Xmax & 22,30 \\
\hline
\end{tabular}

La resistencia a compresión normalizada fb, según norma [4], es de 15,2 N/mm²

En la gráfica adjunta, se muestra la variabilidad de resultados con una regresión cuadrática de 0.62 , alejada de un valor óptimo de 0.95; es evidente que, con un solo lote de fabricación, la dispersión obtenida es más alta de lo esperado, pero se observan los valores de resistencia a compresión comparables con un hormigón H17.5-H20 


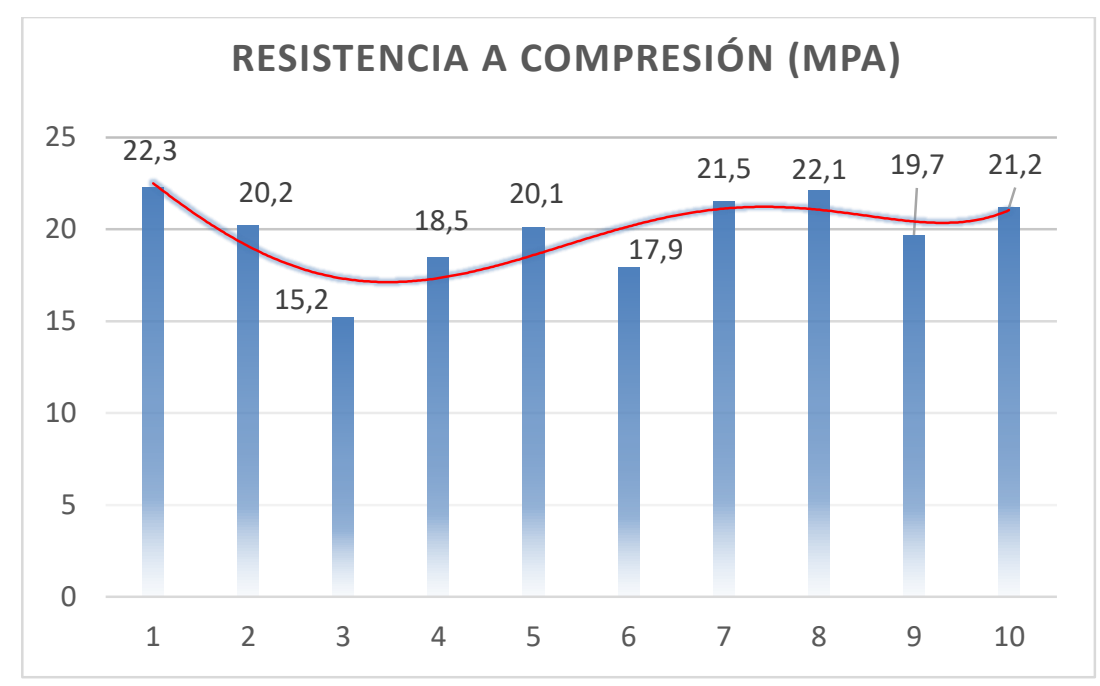

Figura 2: Representación de resultados.

Un ladrillo cerámico de similares características, cierra su resistencia mecánica en valores de $10 \mathrm{~N} / \mathrm{mm}^{2}$; como se observa, valores muy inferiores a los obtenidos en los ensayos.
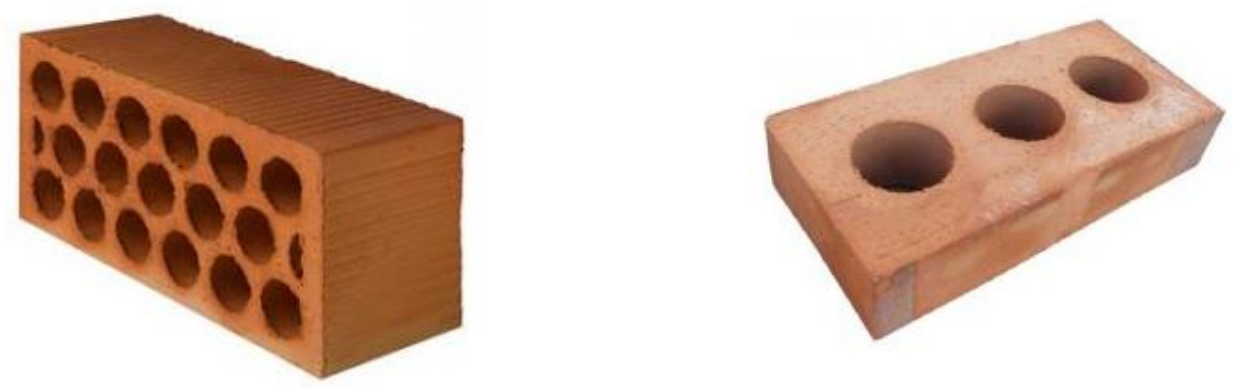

Figura 3: Ladrillos semimacizos de arcilla. (Foto extraída de La plataforma de la construcción).

\subsection{Resultados y Discusión estudio energético.}

El estudio parte de un supuesto de fabricación con dos máquinas que producen $20 \mathrm{t} / \mathrm{h}$. la producción total anual, es estimada para un solo turno de trabajo de 8 horas, en 70.400 t/año.

Se aclara que la tecnología de fabricación por extrusión de los ladrillos cerámicos, difiere de la considerada en el sistema de vibro-compresión, ya que la producción del primer caso, establece ratios mayores de ladrillos/hora. Pero, se indica que el consumo de las extrusoras supera muy mucho el de las prensas hidráulicas. En este sentido, el análisis de consumo eléctrico/producción, aunque no se refleja en el documento, tiene cierto paralelismo quedando a favor la tecnología de prensando. Un estudio de fabricación, entre otros, lo encontramos en plan de negocio de una ladrillera [2], y el de consumo eléctrico y térmico, el documento de análisis de factividad de una fábrica de ladrillos [3], y del trabajo de Auditoría energética de una industria cerámica de fabricación de tejas y ladrillos [6] de Ruiz de la Cruz.

El problema del ladrillo cerámico es que la fabricación, se establece en dos fases separadas (una vez fabricado el ladrillo). La primera corresponde al secado antes de la cocción.

No existe una sola forma de secar y dependerá del tiempo y la temperatura, aunque, en cualquier caso, no puede superarse $\operatorname{los} 100^{\circ} \mathrm{C}$

Si suponemos esa temperatura de consigna que provocará la evaporación del agua de un modo lento, establecido en un periodo de 8 horas, y como unidad de consumo establecemos 


\section{CONGRESO IBÉRICO DE AGROINGENIERÍA \\ X CONGRESSO IBÉRICO DE AGROENGENHARIA \\ 3 - 6 septiembre 2019, Huesca - España}

$90.000 \mathrm{kcal} / \mathrm{t}$; tenemos que, para la producción indicada, el consumo será de 50.688.000.000 $\mathrm{kcal} / \mathrm{año}$ que por 4.18 , tendremos $211.875 .840 .000 \mathrm{KJ} / \mathrm{año}$

Si se define una tonelada equivalente de petróleo, o energía que es capaz de rendir $1 \mathrm{t}$ de petróleo (tep, o toe) como $41.868 .000 \mathrm{KJ}$, o $11.630 \mathrm{KW} / \mathrm{h}$, tenemos para esa energía estimada, el valor de 5.061 tep

Los factores de conversión, se han obtenido de IDAE [5], siendo, como referencia y ejemplo, para el carbón para coque, de 4,40 tCO2/tep.

Si continuamos con el proceso, una vez seco el ladrillo, viene la fase de cocción. Es variable del mismo modo que en el caso anterior y, consultados distintos fabricantes, la metodología cambia, si bien la estimación puede ser la siguiente: temperatura de cocción $900^{\circ} \mathrm{C}$ durante 8 horas.

La unidad de consumo (extraída de un balance sencillo de energía), es de $1.050 .000 \mathrm{Kcal} / \mathrm{t}$ lo que lleva a un consumo para la fabricación indicada de 70400t/año de $591.360 .000 \mathrm{Kcal} / \mathrm{año}$; 2.471.884.800.400KJ/año y 59.040 tep.

En cuanto al ladrillo de yeso, el secado se hace al aire, por tanto, la energía a considerar en el proceso de fabricación es la del propio yeso.

Con una temperatura de $180^{\circ} \mathrm{C}$ se puede obtener yeso. Solo hay que extraer la molécula de agua para convertirlo en yeso dihidrato. En el balance, vamos a subir la temperatura a $250^{\circ} \mathrm{C}$ y 1 hora de cocción. El consumo estimado del balance será de $270.000 \mathrm{kcal} / \mathrm{t}(\mathrm{Q}=1000 \mathrm{~kg} \times 1.2 \times(250-$ 25)). Para la producción indicada: $243.000 .000 \mathrm{Kcal} / \mathrm{año} ; 1.015 .740 .000 \mathrm{KJ} / \mathrm{año} \mathrm{y}$ un tep de 24 .

La diferencia de ambos procesos, es de 64.076 tep con una reducción de 185.821 tCO2/año para la producción establecida.

\section{Conclusiones}

Se consigue un ladrillo perforado de resistencia a compresión normalizada según [5], de 15,2 $\mathrm{N} / \mathrm{mm}^{2}$, valor muy superior al ladrillo cerámico de similares características.

Se consigue una reducción en la fabricación de emisiones de CO2 con el ladrillo de sulfato cálcico, de más del $80 \%$ en proporción directa. Como referencia para una producción de 70400t/año, la reducción es de 185.821 tCO2.

\section{Referencias}

1. Norma EN UNE 13279-1. 2006: Yesos para la construcción. Parte 1: definiciones y especificaciones.

2. Buitrago Caicedo, Mónica. Universidad de Nariño, Año 2015. Plan de negocio para la creación de una ladrillera tipo refractarios en el municipio de San Andrés de Tumaco.

3. Guillen Coello, Hernan. Universidad Politécnica Salesiana de Ecuador. 2011. Análisis de Factividad para la Implemetnación de una Fábrica de Ladrillos para la Construcción.

4. Norma UNE-EN 772-1 + A1, junio 2016. Métodos de ensayo para fábricas de albañilería. Parte 1. Determinación de la resistencia a compresión.

5. Instituto para la Divulgación y Ahorro de Energía. IDEA. Ministerio de Industria, Turismo y Comercio. Factores de conversión energía final-energía primaria y factores de emisión de CO2. Año 2010.

6. Ruiz De La Cruz, D. (2016). Auditoría energética de una industria cerámica de fabricación de tejas y ladrillos. http://hdl.handle.net/10251/72725. Universidad Politécnica de Valencia. 